\title{
On Born approximation in black hole scattering
}

\author{
D. Batic ${ }^{1}$, N. G. Kelkar ${ }^{2}$ and M. Nowakowski ${ }^{2}$ \\ 1 Department of Mathematics, University of West Indies, Kingston 6, Jamaica \\ 2 Departamento de Fisica, Universidad de los Andes, \\ Cra.1E No.18A-10, Bogota, Colombia
}

\begin{abstract}
A massless field propagating on spherically symmetric black hole metrics such as the Schwarzschild, Reissner-Nordström and Reissner-Nordström-de Sitter backgrounds is considered. In particular, explicit formulae in terms of transcendental functions for the scattering of massless scalar particles off black holes are derived within a Born approximation. It is shown that the conditions on the existence of the Born integral forbid a straightforward extraction of the quasi normal modes using the Born approximation for the scattering amplitude. Such a method has been used in literature. We suggest a novel, well defined method, to extract the large imaginary part of quasinormal modes via the Coulomb-like phase shift. Furthermore, we compare the numerically evaluated exact scattering amplitude with the Born one to find that the approximation is not very useful for the scattering of massless scalar, electromagnetic as well as gravitational waves from black holes.
\end{abstract}

PACS numbers: 04.70.-s, 03.65.Nk, 04.62.+v 


\section{INTRODUCTION}

The theory of the scattering of massless and massive particles with different spins off black holes has a long history in gravity and quantum gravity [1]. Over the years the topic attracted much attention and led to the discovery of new phenomena like glory scattering [2], superradiant scattering and black hole bomb [3]. A related subject is the modes of perturbation of black holes (characteristic sound of a black hole), the so-called Quasi Normal Modes (QNMs) [4], which can be also extracted from the corresponding scattering $S$-matrix where they appear as complex poles (resonances). The relevance of QNMs for the quantum gravity lies, e.g., in a method to quantize the area (and therefore also the entropy) of a black hole. However, the ramification of the $S$-matrix poles goes beyond the QNMs as modes of perturbation. The poles normally harbour all the information about the existence of stable bound states, quasi-bound states and resonances [5]. For instance, the absence of a stable bound state of a particle in a black hole metric [6] must get reflected in the properties of the poles of the $S$-matrix which in such a case should always have a nonzero imaginary part. For massless fields the scattering amplitude has, in addition, a cut in the complex frequency plane beginning from the zero frequency. The lack of bound state of a spin-1/2 particle in certain black hole spacetimes [6] indicates a non-local quantum effect in semi-classical black hole physics since the classical picture yields a stable orbit. It is therefore not surprising that different mathematical methods have been applied to the theory of black hole scattering to probe into the semi-classical aspects of quantum gravity [7-9]. Recently a suggestion to extract QNMs from the Born approximation to the scattering amplitude was made in [10-12] with apparently reasonable results for the imaginary part of the QNMs. It is often tempting to use such simplified approximations to obtain analytical results. In [13] it was shown that the extraction of QNMs from the Born amplitude for the Schwarzschild and Schwarzschild de Sitter case is mathematically not well founded. In

the second reference in [13], a regularization method to evaluate the otherwise divergent integral was suggested. However, the fact remains that the Born integral simply does not exist for a certain range of the energy parameter and it does not make sense to insist on extracting poles from such an integral. Here we will demonstrate that this feature is more general and that the Born integrals for all interesting spacetimes, including ReissnerNordström and Reissner-Nordström de Sitter exist only within a restricted validity range of 
the energy $k \equiv \omega$ which lies outside the range of the extracted values of the QNMs. This is in agreement with the observation that there are no $S$-matrix poles in the Born approximation of the Feynman-Dyson perturbative expansion. In an attempt to remedy this problem, we prove that the large imaginary parts of the QNMs can be obtained in a mathematically rigorous way through the Coulomb-like scattering phase. Secondly, it appears timely to make an explicit check of the limitations of the Born approximation in the physical region of the phase space, i.e., its usefulness to describe correctly the scattering process. Some work in this direction has been already done in [14, 15]. However, either the distorted wave Born approximation is used [15] or the comparison with the Born approximation is done for low frequencies and in terms of the differential cross section $d \sigma / d \Omega$ paying attention to the comparison using scattering angles. We perform a test of the validity of the Born approximation for different $l$ and in the case of the scattering of massless fields with spin $s=0$, 1, 2. There also exists an ambiguity in the scattering phase shift in the Born approximation which appears in literature [16]. Hence there arises a general question whether it is reasonable to apply the Born approximation for the scattering off black holes. We therefore make a comparison between exact albeit numerical results obtained via a Riccati equation for the reflection coefficient $\mathcal{R}$ (in the context of a one dimensional tunneling problem into which we can map the scattering problem) and the Born approximation. The comparison shows that only for the spin 0 case and the $l=0$ partial wave, the agreement between the exact result and the Born approximation is reasonable over a wide range of $\omega$. For spins, $s=1,2$, there is no agreement for any $l$.

It is well known from general scattering theory [17] that the Born approximation might work at high energies [17]. However, if the required energies are so high that the reflection coefficient goes to zero, the comparison might lose any physical meaning. There exist circumstances where the Born approximation is expected to be bad and then again circumstances where it is valid for some particular angular momentum $l$ provided we mean the Born approximation for a partial wave [17]. This is what happens at least in the case of $s=0$.

The article is organized as follows. We briefly outline the formalism in section two. Section three is about the possibility to extract the QNMs from the Born amplitude. In section four we obtain an alternative way to obtain the QNMs. In section five we compare the exact scattering amplitude results with those in the Born approximation. In section six 
we draw our conclusions.

\section{BLACK HOLE SCATTERING AND THE BORN AMPLITUDE}

We consider the propagation of a massless scalar field $\phi=\phi(t, r, \vartheta, \varphi)$ governed by the wave equation $g^{\mu \nu} \nabla_{\mu} \nabla_{\nu} \phi=0$ where $g_{\mu \nu}$ denotes a static, spherically symmetric black hole metric whose line element is

$$
d s^{2}=f(r) d t^{2}-\frac{d r^{2}}{f(r)}-r^{2}\left(d \vartheta^{2}+\sin ^{2} \vartheta d \varphi^{2}\right) .
$$

Taking into account that the eigenvalues of the spherical Laplacian are $-\ell(\ell+1)$ the wave equation can be separated by means of the ansatz

$$
\phi(t, r, \vartheta, \varphi)=e^{i \omega t} \frac{1}{r} \psi_{n \ell \omega}(r) Y_{\ell m}(\vartheta, \varphi), \quad \operatorname{Re}(\omega)>0
$$

giving rise to the Schrödinger-like equation for the radial part

$$
\left[-\frac{d^{2}}{d r_{*}^{2}}+V(r)\right] \psi_{n \ell \omega}=\omega^{2} \psi_{n \ell \omega}
$$

where $V(r)=f(r) U(r)$ (with $f(r)$ having a different form for the various metrics considered in the next section) and

$$
U(r)=\frac{\ell(\ell+1)}{r^{2}}+\frac{f^{\prime}(r)}{r}
$$

Here, a prime denotes differentiation with respect to $r$ whereas $r_{*}$ is a tortoise coordinate defined through

$$
\frac{d r_{*}}{d r}=f(r)^{-1}
$$

We recall that for static, spherically symmetric black hole metrics which are asymptotically flat at spatial infinity the quasinormal modes boundary condition requires that there is a purely ingoing plane wave at the event horizon $r_{0}$ and a purely outgoing plane wave at space-like infinity, i.e.

$$
\psi_{n \ell \omega}(r) \propto \begin{cases}e^{i \omega r_{*}} & \text { if } r_{*} \rightarrow-\infty, \\ e^{-i \omega r_{*}} & \text { if } r_{*} \rightarrow+\infty,\end{cases}
$$

whereas for black hole metrics which are asymptotically de Sitter at spatial infinity the appropriate boundary condition for analyzing quasinormal modes is that the wave function should be outgoing at the cosmological horizon $r_{c}$ but vanishing at the origin [10]

$$
\psi_{n \ell \omega}(r) \propto \begin{cases}0 & \text { if } r \rightarrow 0, \\ e^{-i \omega r_{*}} & \text { if } r \rightarrow r_{c} .\end{cases}
$$


Quasinormal modes can be found by computing the poles of the scattering amplitude $S(\omega)$. To see that we write the boundary condition at $r_{*} \rightarrow \infty$ or $r \rightarrow r_{c}$ in the form

$$
A(\omega) e^{+i \omega r_{*}}+B(\omega) e^{-i \omega r_{*}} \propto\left(e^{+i \omega r_{*}}+S(\omega) e^{-i \omega r_{*}}\right)
$$

where $S(\omega)$ is a component of the scattering matrix $\mathbf{S}$. Hence imposing $A(\omega)=0$ is equivalent to looking for a subset of $S$-matrix poles. Since the problem of black hole scattering is mapped into one dimension it makes sense to make some identifications. The one dimensional problem is equivalent to a quantum mechanical tunneling problem such that the reflection amplitude $R(\omega)$ is simply identical to $S(\omega)$. In one dimension (1D) $S(\omega)$ is thus identified with the scattering amplitude $f^{1 \mathrm{D}}(\omega)$ via $S(\omega)=R(\omega)=i f^{1 \mathrm{D}}(\omega) / 2 \omega[18$ ]. This is different from the identification in three dimensions $(3 \mathrm{D})$ where $S(\omega)=1+2 i \omega f^{3 \mathrm{D}}(\omega)$. In literature, while evaluating cross sections, considering the asymptotic behaviour of the wave function in the 3D radial coordinate $r$, generally $R(\omega)$ is identified with $S(\omega)=e^{2 i \delta(\omega)}$ [19] where $\delta(\omega)$ is the scattering phase shift. The reflection and transmission in 1D can be identified as two channels in a scattering problem such that the $R(\omega)$ and $T(\omega)$ enter the elastic and absorption cross sections respectively. The unitarity of the $S$-matrix, $\mathbf{S}^{\dagger} \mathbf{S}=1$, is then given by $|R(\omega)|^{2}+|T(\omega)|^{2}=1$. We shall discuss this in more detail in section IV.

In one dimension the Born approximation for the scattering amplitude is given by [10]

$$
f_{\text {Born }}^{1 D}(\omega)=\int_{-\infty}^{+\infty} d r_{*} V\left(r_{*}\right) e^{2 i \omega r_{*}} .
$$

In particular, for static, spherically symmetric black hole metrics which are asymptotically flat, the scattering amplitude reads

$$
f_{B o r n}^{1 D}(\omega)=\int_{r_{0}}^{+\infty} d r U(r) e^{2 i \omega r_{*}(r)},
$$

whereas for those black hole backgrounds going over asymptotically at spatial infinity to a de Sitter geometry we have

$$
f_{B o r n}^{1 D}(\omega)=\int_{r_{0}}^{r_{c}} d r U(r) e^{2 i \omega r_{*}(r)} .
$$

Some comments are now in order. A first remark refers to the nature of the two channel process which indicates that the phase shift $\delta(\omega)$ cannot be real in general. Indeed, we have $S(\omega)=e^{2 i \delta(\omega)}=e^{-2 \operatorname{Im} \delta(\omega)} e^{2 i \operatorname{Re} \delta(\omega)}=\eta e^{2 i \operatorname{Re} \delta(\omega)}$ where $\eta$ is the inelasticity factor. If the reflection amplitude is identified with $S(\omega)$, any usage of an approximation where the 
phase shift comes out real necessarily neglects the transmission. For instance, this applies to a formula like Eq. (35) for the Born phase shift mentioned in [16]. A second remark concerns the difference between the Born approximation in one and three dimensions. In three dimensions with a potential depending only on $r$, the Born integral $\int e^{-i \mathbf{q} \cdot \mathbf{r}} V(r) d \mathbf{r}$ is equal to $(-4 \pi / q) \int_{0}^{\infty}(\sin (q r) / r) V(r) r^{2} d r$ and is hence real. The Born amplitude in (7) is complex for a real $V(r)$. This implies that the conclusion that the phase shift in the Born approximation must be small [17] is not stringent here.

\section{QUASINORMAL MODES IN THE BORN APPROXIMATION}

In this section we discuss the Schwarzschild and Schwarzschild-de Sitter cases briefly and concentrate on the existence of quasinormal modes (QNMs) in the Reissner-Nordström and Reissner-Nordström-de Sitter cases applying the Born approximation.

\section{A. Remark on Schwarzschild and Schwarzschild-de Sitter metric}

In [13] it was demonstrated that the Born integrals have a certain range of validity for the above mentioned metrics. Indeed, for the Schwarzschild case, the Born integral is restricted to the following range:

$$
0 \leq \omega_{I}<\frac{1}{4 M}
$$

where $\omega_{I}$ is the imaginary part of the quasinormal mode frequency $\omega=\omega_{R}+i \omega_{I}$, with the real part $\omega_{R}>0$. The Born integral in this case is a linear combination of products of the Gamma function and Whittaker function [13]. the latter possess the following poles:

$$
\omega_{n}=-i n \kappa, \quad \omega_{n}=i n \kappa,
$$

which, however, in view of the restrictions on the Born integral cannot be interpreted as quasinormal modes. The Schwarzschild-de Sitter case is similar and we refer the reader to [13] for further details.

\section{B. Reissner-Nordström metric}

In this case $f(r)=1-2 M / r+Q^{2} / r^{2}$ where $M$ and $Q$ are the mass and charge of the black hole respectively. We shall analyze the scattering amplitude both for the non extreme 
$M>|Q|$ and extreme case $M=|Q|$. In the non extreme case the black hole possesses two distinct horizons at $r_{ \pm}=M \pm \sqrt{M^{2}-Q^{2}}$ whose surface gravity is given by the simple formula

$$
\kappa_{ \pm}=\frac{r_{+}-r_{-}}{2 r_{ \pm}^{2}} .
$$

Notice that since $r_{+}>r_{-}, \kappa_{-}$will always be greater than $\kappa_{+}$. The scattering amplitude is represented by the integral

$$
f_{\text {Born }}^{1 D}(\omega)=\int_{r_{0}}^{+\infty} d r U(r) e^{2 i \omega r_{*}(r)}, \quad U(r)=\frac{\ell(\ell+1)}{r^{2}}+\frac{2 M}{r^{3}}-\frac{2 Q^{2}}{r^{4}} .
$$

Taking into account that we are interested in the region $r>r_{+}$the tortoise coordinate $r_{*}$ will be given by the following expression [23]

$$
r_{*}=r+\frac{1}{2 \kappa_{+}} \ln \left(\frac{r}{r_{+}}-1\right)-\frac{1}{2 \kappa_{-}} \ln \left(\frac{r}{r_{-}}-1\right) .
$$

In view of the above relation it is not difficult to see that we have to evaluate integrals of the form

$$
I_{s}=\int_{r_{+}}^{\infty} d r r^{-s}\left(\frac{r}{r_{+}}-1\right)^{i \omega / \kappa_{+}}\left(\frac{r}{r_{-}}-1\right)^{-i \omega / \kappa_{-}} e^{2 i \omega r}, \quad s=2,3,4 .
$$

By means of the substitution $x=\left(r / r_{+}\right)-1$ the above integral becomes up to a constant multiplicative factor

$$
\mathcal{I}_{s}=\int_{0}^{\infty} d x h(x), \quad h(x)=\frac{x^{i \frac{\omega}{\kappa_{+}}}}{(x+1)^{s}(x+\Delta)^{i \frac{\omega}{\kappa_{-}}}} e^{2 i \omega x}, \quad \Delta=\left(r_{+}-r_{-}\right) / r_{+}>0 .
$$

Taking into account that

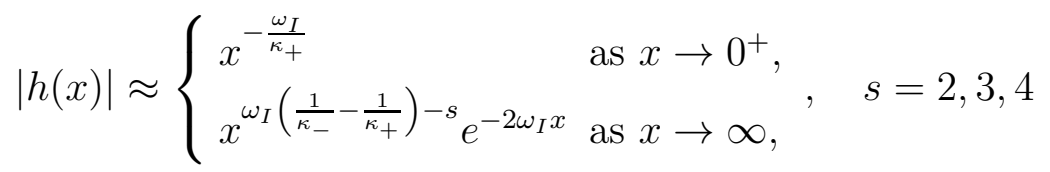

the integral $\mathcal{I}_{s}$ will exist if the imaginary part of the quasinormal frequency is restricted to the following range

$$
0 \leq \omega_{I}<\kappa_{+} .
$$

Let $\omega_{I} \in\left[0, \kappa_{+}\right)$. Unfortunately the above integral cannot be computed in closed form. However, it takes a dominant contribution close to the event horizon $x \approx 0$. Hence, we shall consider the integral

$$
\int_{0}^{\infty} d x x^{i \frac{\omega}{\kappa_{+}}} e^{2 i \omega x}=\frac{i}{2 \omega(-2 i \omega)^{\frac{i \omega}{\kappa_{+}}}} \Gamma\left(1+i \frac{\omega}{\kappa_{+}}\right) .
$$


From this result we immediately see that the imaginary parts of the quasinormal modes will be given by

$$
\omega_{n}=i n \kappa_{+}, \quad n \gg 1 .
$$

However, these values should be disregarded in view of the condition (12). In the extreme case $M=Q$ the event and Cauchy horizon coalesce into a single horizon at $r_{0}=M$. Then, the spatial function $f(r)$ can be rewritten as $f(r)=r^{2} /(r-M)^{2}$, whereas the tortoise coordinate is given by

$$
r_{*}=M\left[\rho-\frac{1}{\rho-1}+\ln (\rho-1)^{2}\right], \quad \rho=\frac{r}{M} .
$$

By means of the above relation and the transformation $x=\rho-1$ the scattering amplitude can be written as

$$
f_{\text {Born }}^{1 D}(\Omega)=\frac{e^{i \Omega}}{M} \int_{0}^{\infty} d x\left[\frac{\ell(\ell+1)}{(x+1)^{2}}+\frac{2}{(x+1)^{3}}-\frac{2 Q^{2}}{M^{2}(x+1)^{4}}\right] x^{2 i \Omega} e^{i \Omega\left(x-\frac{1}{x}\right)},
$$

where $\Omega=2 M \omega$. Essentially we have to solve integrals of the form

$$
\mathcal{I}_{s}=\int_{0}^{\infty} d x \frac{x^{2 i \Omega}}{(x+1)^{s}} e^{i \Omega\left(x-\frac{1}{x}\right)}
$$

with $s=2,3,4$. Taking into account that

$$
\left|\frac{x^{2 i \Omega}}{(x+1)^{s}} e^{i \Omega\left(x-\frac{1}{x}\right)}\right| \approx\left\{\begin{array}{ll}
x^{-2 \Omega_{I}} e^{\frac{\Omega_{I}}{x}} & \text { as } x \rightarrow 0^{+} \Longrightarrow \Omega_{I}<0, \\
x^{-2 \Omega_{I}-s} e^{-\Omega_{I} x} & \text { as } x \rightarrow \infty \Longrightarrow \Omega_{I}>0,
\end{array}, \quad s=2,3,4\right.
$$

the integral $\mathcal{I}_{s}$ has a chance to exist only if $\Omega_{I}=0$. Let $\Omega_{I}=0$ in (13). Since the corresponding integral cannot be computed in closed form, we shall consider only the dominant contribution close to the event horizon $x \approx 0$. Hence, we obtain [20]

$$
\int_{0}^{\infty} d x x^{2 i \Omega_{R}} e^{-i \frac{\Omega_{R}}{x}}=(i \Omega)^{2 i \Omega+1} \Gamma\left(-1-2 i \Omega_{R}\right) .
$$

From this result we immediately see that the poles of the Gamma function should occur at the complex values

$$
\Omega_{R, n}=-\frac{i n}{2}, \quad n \gg 1
$$

which is impossible. In this case too, the Born approach is inconclusive for the determination of the poles of the scattering amplitude. 


\section{Reissner-Nordström-de Sitter metric}

In the present case

$$
f(r)=1-\frac{2 M}{r}+\frac{Q^{2}}{r^{2}}-\frac{\Lambda r^{2}}{3}
$$

where the constants $M>Q, Q \in \mathbb{R}$ are the mass and electric charge of the black hole and $\Lambda \geq 0$ is the cosmological constant. In general, the equation $f(r)=0$ has four different roots: a negative root $r_{1}$ without a physical meaning, a minimum positive root $r_{c}$ corresponding to a Cauchy horizon, a root $r_{-}>r_{c}$ interpreted as an event horizon and a root $r_{c}>r_{-}>r_{c}$ corresponding to a cosmological horizon. According to [25, 26] these horizons are given by

$$
r_{+}=-A+B+C, \quad r_{-}=A-B+C, \quad r_{c}=A+B-C
$$

where

$$
\begin{aligned}
& A=\frac{1}{\sqrt{2 \Lambda}} \sqrt{1-\sqrt{1-4 \Lambda Q^{2}} \cos \left(\frac{\alpha}{3}-\frac{\pi}{3}\right)} \\
& B=\frac{1}{\sqrt{2 \Lambda}} \sqrt{1-\sqrt{1-4 \Lambda Q^{2}} \cos \left(\frac{\alpha}{3}+\frac{\pi}{3}\right)} \\
& C=\frac{1}{\sqrt{2 \Lambda}} \sqrt{1+\sqrt{1-4 \Lambda Q^{2}} \cos \left(\frac{\alpha}{3}\right)}
\end{aligned}
$$

and

$$
\alpha=\arccos \left[-\frac{1-6 \Lambda\left(3 M^{2}-2 Q^{2}\right)}{\left(1-4 \Lambda Q^{2}\right)^{3 / 2}}\right] .
$$

In the exterior region $r_{-}<r<r_{+}$we introduce a tortoise coordinate $r_{*}$ by means of the relation

$$
\frac{d r_{*}}{d r}=\frac{1}{f(r)} .
$$

Taking into account that the surface gravities at the the three horizons are given by [27]

$$
\begin{aligned}
\kappa_{+} & =\frac{\Lambda}{6 r_{+}^{2}}\left(r_{+}-r_{1}\right)\left(r_{+}-r_{c}\right)\left(r_{+}-r_{-}\right), \\
\kappa_{-} & =\frac{\Lambda}{6 r_{-}^{2}}\left(r_{-}-r_{1}\right)\left(r_{-}-r_{c}\right)\left(r_{+}-r_{-}\right), \\
\kappa_{c} & =\frac{\Lambda}{6 r_{c}^{2}}\left(r_{c}-r_{1}\right)\left(r_{-}-r_{c}\right)\left(r_{+}-r_{c}\right),
\end{aligned}
$$

the tortoise coordinate is explicitly given by

$$
r_{*}=\frac{1}{2 \kappa_{-}} \ln \left|\frac{r}{r_{-}}-1\right|-\frac{1}{2 \kappa_{+}} \ln \left|1-\frac{r}{r_{+}}\right|-\frac{1}{2 \kappa_{c}} \ln \left|\frac{r}{r_{c}}-1\right|+\frac{1}{a} \ln \left|\frac{r}{r_{1}}-1\right|
$$


where

$$
a=\frac{\Lambda}{6 r_{1}^{2}}\left(r_{c}-r_{1}\right)\left(r_{-}-r_{1}\right)\left(r_{+}-r_{1}\right)
$$

Hence, the scattering amplitude is given by

$$
f_{\text {Born }}^{1 D}(\omega)=\int_{r_{-}}^{r_{+}} d r U(r)\left(\frac{r}{r_{-}}-1\right)^{i \frac{\omega}{\kappa_{-}}}\left(1-\frac{r}{r_{+}}\right)^{-i \frac{\omega}{\kappa_{+}}}\left(\frac{r}{r_{c}}-1\right)^{-i \frac{\omega}{\kappa_{c}}}\left(\frac{r}{r_{1}}-1\right)^{2 i \omega a}
$$

with

$$
U(r)=\frac{\ell(\ell+1)}{r^{2}}+\frac{2 M}{r^{3}}-\frac{2 Q^{2}}{r^{4}}-\frac{2}{3} \Lambda .
$$

By means of the transformation $u=\left(r-r_{-}\right) /\left(r-r_{+}\right)$mapping the event horizon to 0 and the cosmological horizon to 1 we can reduce the computation of $f_{B o r n}^{1 D}(\omega)$ to the computation of the following integral

$$
\mathcal{I}_{s}=\int_{0}^{1} d u G_{s}(u), \quad s=0,2,3,4
$$

where

$$
G_{s}(u)= \begin{cases}\frac{u^{i \frac{\omega}{\kappa_{-}}}(1-u)^{-i \frac{\omega}{\kappa_{+}}}}{(1-y u)^{s}(1-\Xi u)^{i \frac{\omega}{\kappa_{c}}}(1-w u)^{2 i \omega a}} & \text { for } s=2,3,4, \\ \frac{u^{i \frac{\omega}{\kappa_{-}}}(1-u)^{-i \frac{\omega}{\kappa_{+}}}}{(1-\Xi u)^{i \frac{\omega}{\kappa_{c}}}(1-w u)^{2 i \omega a}} & \text { for } s=0,\end{cases}
$$

with $y$

$$
\Xi=\frac{r_{-}-r_{+}}{r_{-}-r_{c}}, \quad w=\frac{r_{-}-r_{+}}{r_{-}-r_{1}}, \quad y=\frac{r_{-}-r_{+}}{r_{-}} .
$$

Taking into account that

$$
\left|G_{s}(u)\right| \approx\left\{\begin{array}{ll}
u^{-\frac{\omega_{I}}{\kappa_{-}}} \quad \text { as } u \rightarrow 0^{+} \Longrightarrow \omega_{I}<\kappa_{-}, \\
(1-u)^{\frac{\omega_{I}}{\kappa_{+}}} \text {as } u \rightarrow 1^{-} \Longrightarrow \omega_{I}>-\kappa_{+},
\end{array}, \quad s=0,2,3,4\right.
$$

it follows that the above integral exists if and only if the imaginary part of the quasinormal mode satisfies the condition

$$
-\kappa_{+}<\omega_{I}<\kappa_{-} .
$$

Fortunately, the integral $\mathcal{I}_{s}$ can be computed in closed form in terms of Gamma functions and the Lauricella function $F_{D}^{(n)}$ with $n=3$ whose Picard's integral representation is given by [28, 29]

$$
\int_{0}^{1} d u u^{a-1}(1-u)^{c-a-1} \prod_{i=1}^{n}\left(1-x_{i} u\right)^{-b_{i}}=\frac{\Gamma(a) \Gamma(c-a)}{\Gamma(c)} F_{D}^{(n)}\left(a, b_{1}, b_{2}, \cdots, b_{n} ; c ; x_{1}, x_{2}, \cdots, x_{n}\right)
$$

with

$$
F_{D}^{(n)}\left(a, b_{1}, b_{2}, \cdots, b_{n} ; c ; x_{1}, x_{2}, \cdots, x_{n}\right)=\sum_{i_{1}, i_{2}, \cdots, i_{n}=0}^{\infty} \frac{(a)_{i_{1}+i_{2}+\cdots+i_{n}}\left(b_{2}\right)_{i_{2}} \cdots\left(b_{n}\right)_{i_{n}}}{(c)_{i_{1}+i_{2}+\cdots+i_{n}} i_{1} ! i_{2} ! \cdots i_{n} !} x_{1}^{i_{1}} x_{2}^{i_{2}} \cdots x_{n}^{i_{n}}
$$


absolutely convergent for $\max \left\{\left|x_{1}\right|,\left|x_{2}\right|, \cdots,\left|x_{n}\right|\right\}<1$. An analysis similar to that performed in [10] shows that the poles of the scattering amplitude are located at

$$
\omega_{n}=i n \kappa_{-}, \quad \omega_{n}=-i n \kappa_{+} \quad n \gg 1
$$

but we should disregard them in virtue of the integrability condition (14).

\section{QUASINORMAL MODES FROM A COULOMB-LIKE PHASE SHIFT}

For large $r$ one can expect that the scattering can be described by a $1 / r$ Newtonian potential. Indeed, in [30], such an analysis has been explicitly performed. Starting with the Klein-Gordon equation, $\square \Psi=g_{\mu \nu} \nabla^{\mu} \nabla^{\nu} \Psi=0$ and using the separation ansatz,

$$
\Psi(t, r, \theta, \phi)=T(t) R(r) \Theta(\theta, \phi)
$$

the transformation

$$
R=u(r)[r(1-2 M / r)]^{-1 / 2}
$$

reveals the asymptotic form,

$$
R \stackrel{r \rightarrow \infty}{\longrightarrow}(1 / r) \sin \left[\omega r-\gamma \ln (2 \omega r)-(1 / 2) l \pi-\sigma_{l}\right]
$$

where $\gamma=-2 M \omega$ and $\sigma_{l}$ is the equivalent Coulomb phase shift given by

$$
\sigma_{l}=\arg \Gamma(l+1-2 i M \omega)
$$

Using the property $\Gamma(\bar{z})=\overline{\Gamma(z)}$, the partial wave scattering matrix is given by

$$
e^{2 i \sigma_{l}}=\frac{\Gamma(l+1-2 i M \omega)}{\Gamma(l+1+2 i M \omega)} .
$$

The poles corresponding to the above amplitude are $\omega_{n}=-i \kappa 2(n+l+1)$ which for large $n$ can be written as $\omega_{m}=-i \kappa m$. It therefore appears that the large imaginary parts of the QNM frequencies are nothing but the poles of the Coulomb-like amplitude.

Two comments are in order. Let us first note that in [30] the signature of the metric is $(1,-1,-1,-1)$ and therefore the time development is proportional to $\exp (-i \omega t)$. With $\omega=$ $-i \kappa m$, this corresponds to a decaying state $\exp (-\kappa m t)$. Physically this case is equivalent to working with a signature $(-1,1,1,1)$ and the time evolution $\exp (+i \omega t)$ with the QNMs given by $\omega=+i \kappa m$. Secondly we remark that in atomic physics the definition of the principal quantum number is $n=j_{\max }+l+1$ which is very similar to the definition of $m$ above. 


\section{VARIABLE AMPLITUDE METHOD FOR BLACK HOLE SCATTERING}

The Schrödinger like equation (2) in black hole scattering can be solved using standard techniques for tunneling in quantum mechanics. The asymptotic solutions of the Schrödinger equation (2) are:

$$
\begin{aligned}
& \psi=A(\omega) e^{+i \omega r_{*}}+B(\omega) e^{-i \omega r_{*}}, \quad r_{*} \rightarrow-\infty \\
& \psi=C(\omega) e^{+i \omega r_{*}}+D(\omega) e^{-i \omega r_{*}}, \quad r_{*} \rightarrow+\infty .
\end{aligned}
$$

For waves incident on the black holes from the right (i.e. $+\infty), B(\omega)=0$, the reflection amplitude $R(\omega)=D(\omega) / C(\omega)$ and the transmission amplitude $T(\omega)=A(\omega) / C(\omega)$, so that

$$
\begin{gathered}
\psi=T(\omega) e^{i \omega r_{*}}, \quad r_{*} \rightarrow-\infty \\
\psi=e^{i \omega r_{*}}+R(\omega) e^{-i \omega r_{*}}, \quad r_{*} \rightarrow+\infty .
\end{gathered}
$$

The reflection amplitude $R(\omega)$ is related to the scattering amplitude $f^{1 D}(\omega)$ in one dimension (1D) as $R(\omega)=i f^{1 D}(\omega) / 2 \omega$, where

$$
f^{1 D}(\omega)=\int_{-\infty}^{\infty} e^{i \omega x} V(x) \psi(x) d x
$$

and reduces to (77) in the Born approximation. We shall evaluate $R(\omega)$ numerically via the variable amplitude method. This $R(\omega)$ corresponds to the exact scattering amplitude $f^{1 D}(\omega)$ and will be compared with $R^{B o r n}(\omega)=i f_{B o r n}^{1 D}(\omega) / 2 \omega$. The variable amplitude method was first introduced in [31] and has been widely used to evaluate the reflection and transmission coefficients for different potentials in literature [32].

The scattering matrix in $1 \mathrm{D}$ is given as

$$
\mathbf{S}=\left(\begin{array}{cc}
T_{L}(\omega) & R_{R}(\omega) \\
R_{L}(\omega) & T_{R}(\omega)
\end{array}\right)
$$

where $R_{L}(\omega), T_{L}(\omega)$ are the reflection and transmission amplitudes respectively for incidence from the left $(-\infty)$ and $R_{R}(\omega), T_{R}(\omega)$ for incidence from the right $(+\infty)$. For elastic scattering, $T_{L}(\omega)=T_{R}(\omega)$. Though the amplitudes for left and right incidence may not necessarily be equal, the reflection and transmission coefficients are the same, i.e., $\left|R_{L}(\omega)\right|^{2}=$ $\left|R_{R}(\omega)\right|^{2}=\mathcal{R}(\omega)$ and $\left|T_{L}(\omega)\right|^{2}=\left|T_{R}(\omega)\right|^{2}=\mathcal{T}(\omega)$. Starting with incidence from the right (and dropping the subscript $R$ for convenience), we follow the standard procedure [32] to

obtain the Riccati equation for black hole scattering. This involves writing the solution of 
the Schrödinger equation as a superposition of the reflected and transmitted waves, namely, $\psi\left(\omega, r_{*}\right)=T\left(\omega, r_{*}\right)\left[e^{i \omega r_{*}}+R\left(\omega, r_{*}\right) e^{-i \omega r_{*}}\right]$, which leads to the following equation for $R$ :

$$
\frac{d R\left(\omega, r_{*}\right)}{d r_{*}}=-\frac{V\left(r_{*}\right)}{2 i \omega}\left[e^{i \omega r_{*}}+R\left(\omega, r_{*}\right) e^{-i \omega r_{*}}\right]^{2}
$$

The absence of reflection behind the potential at $r_{*} \rightarrow-\infty$ imposes the boundary condition $R(\omega,-\infty)=0$ on the above equation. The reflection coefficient $\mathcal{R}(\omega)$ is given by $\mathcal{R}(\omega)=$ $|R(\omega, \infty)|^{2}$. Solving the above equation (22) can be made easier by introducing a new function $U\left(\omega, r_{*}\right)$ such that

$$
R\left(\omega, r_{*}\right)=\exp \left(-2 i \omega r_{*}\right)\left[2 i \omega U\left(\omega, r_{*}\right)-1\right] .
$$

The equation for $U$ is,

$$
\frac{d U\left(\omega, r_{*}\right)}{d r_{*}}=1-2 i \omega U\left(\omega, r_{*}\right)-V\left(r_{*}\right) U^{2}\left(\omega, r_{*}\right)
$$

Eq. (24) is solved numerically with the boundary condition $U(\omega,-\infty)=1 / 2 i \omega$ to determine $\mathcal{R}(\omega)=|R(\omega, \infty)|^{2}$.

In Fig. 1 we compare the reflection coefficient obtained by numerically solving the Riccati equation with that obtained within the Born approximation, namely, $\left|R^{\text {Born }}(\omega)\right|^{2}=$ $\left|i f_{\text {Born }}^{1 D}(\omega) / 2 \omega\right|^{2}$. The formalism of section II can be generalized in the Schwarzschild case to arbitrary spin $s$ of the scattered particle. The Regge-Wheeler potential entering the Schrödinger equation is

$$
V\left(r_{*}\right)=\left(1-\frac{2 M}{r}\right)\left[\frac{l(l+1)}{r^{2}}+\frac{2 M\left(1-s^{2}\right)}{r^{3}}\right]
$$

where, $l \geq s$. The Born approximation in general is not expected to be very useful at low energies. However, we find from Fig. 1 that it does not agree even qualitatively with the exact result for any of the three cases considered. One can also see that the reflection coefficient at large energies is very small and any agreement (if at all) with the Born amplitude at high energies becomes irrelevant.

\section{SUMMARY}

The Born approximation for particle scattering from black holes has been studied in detail for the most relevant black hole spacetimes. In summary we can say: 

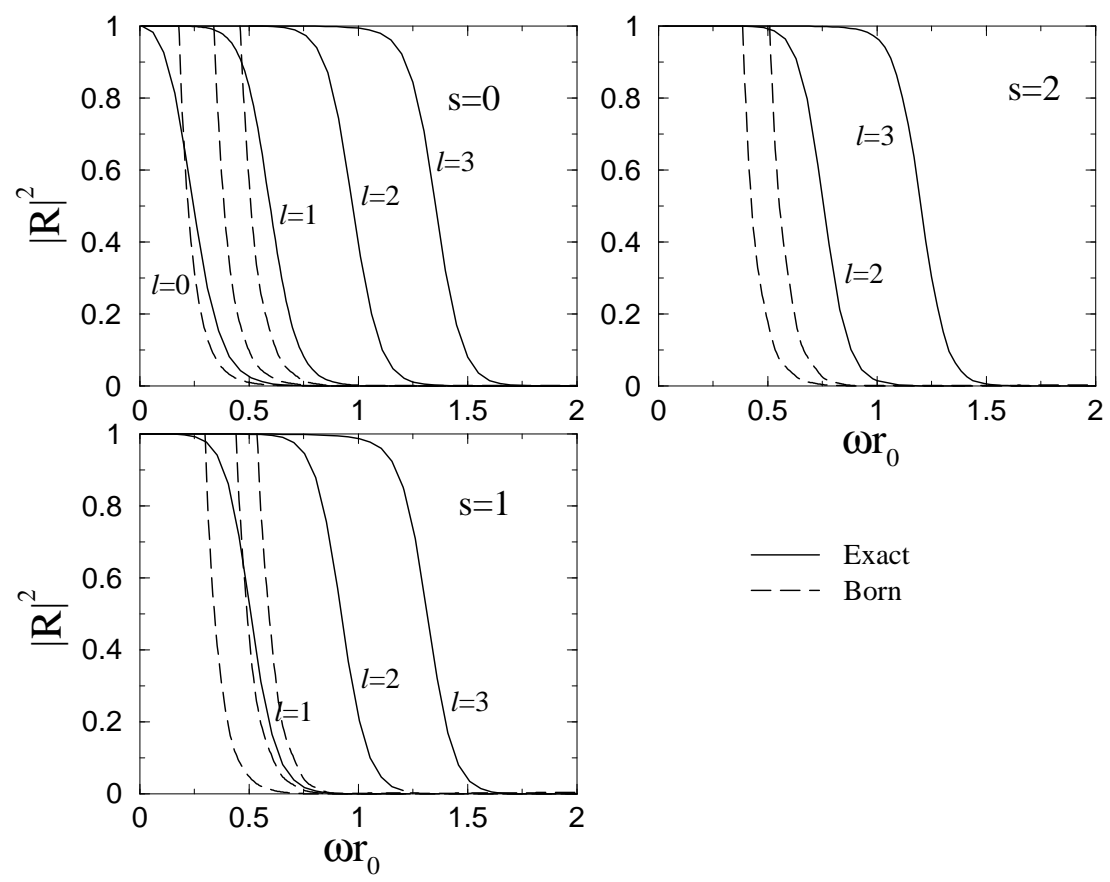

FIG. 1: Reflection coefficient or the scattering amplitude squared for the scattering of massless scalar $(s=0)$, electromagnetic $(s=1)$ and gravitational $(s=2)$ waves from a black hole. The solid lines give the exact results for different $l$. The dashed lines represent the Born approximation results and follow the same sequence for $l$ from left to right.

1. We succeeded in writing the integral of the scattering amplitude in the Born approximation in terms of special functions. The quasinormal modes can in principle be extracted from this parametrization.

2. The conditions for the existence of the Born integral impose a validity range on $\omega$. In all cases of black hole spacetimes we find that the quasinormal modes found here as well as elsewhere in literature using the Born approximation lie outside the validity range. Therefore, it is mathematically impossible to find the quasinormal modes within the Born approximation.

3. We have shown that the quasinormal modes with a large imaginary part can be obtained from the Coulomb-like phase shift in black hole scattering.

4. Comparing the reflection coefficient within the Born approximation with the exact one evaluated numerically (for the case of Schwarzschild black hole) reveals that the only reasonable agreement occurs for the $s=0, l=0$ case. 
[1] N. Sanchez 1978 Absorption and emission spectra of a Schwarzschild black hole, Phys. Rev. D18, 1030 (1978); ibid, Elastic scatttering of waves by a black hole, Phys. Rev D18, 1798 (1978); J. A. H. Futterman, F. A. Handler and R. A. Matzner, Scattering from Black Holes, Cambridge University Press, 1988; N. Anderson and B. Jensen, Scattering by Black Holes in Scattering: scattering and inverse scattering in pure and applied science, Academic Press 2002 .

[2] R. A. Matzner, C. DeWitte-Morette, B. Nelson and T.-R. Zhang, 1985 Glory scattering by black holes, Phys. Rev. D31, 1869.

[3] A. A. Starobinsky, 1973, Sov. Phys.-JETP 37, 28 (1973); A. A. Starobinsky and S. M. Churilov, Sov. Phys.-JETP 38, 1 (1974); W. H: Press and S. A. Teukolsky, 1972 Floating orbits, superradiant scattering and the black hole bomb, Nature 238, 211.

[4] For reviews and more literature see: E. Berti, 2004 Black hole quasinormal modes: hints of quantum gravity?, Arxiv: gr-qc/0411025, presented in Workshop Dynamics and Thermodynamics of Black Holes and Naked Singularities, Milan 2004; H.-P. Nollert, 1999 Quasinormal modes: the characteristic 'sound' of black holes and neutron stars, Class, Quant. Grav. 15, R159; K. D. Kokkotas, 1999 Quasi-Normal modes of stars and black holes, Living Rev. Relativity 2.

[5] R. J. Eden, P. V. Landshoff, D. I. Olive and J. C. Polinghorne, The Analytic S-Matrix, Cambridge University Press, 1966.

[6] A. Lasenby, C. Doran, J. Pritchard, A. Caceres and S. Dolan, 2005 Bound states and decay times of fermions in a Schwarzschild black hole background, Phys. Rev. D72, 105014; M. Winklmeier and O. Yamada, 2006 Spectral analysis of radial Dirac operators in the KerrNewman metric and its applications to time-periodic solutions J. Math. Phys. 47, 102503; D. Batic and M. Nowakowski, 2008 On the bound states of the Dirac equation in the extreme Kerr metric, Class. Quant. Grav. 25, 225022.

[7] E. W. Leaver, 1986 Spectral decomposition of the perturbation response of the Schwarzschild geometry, Phys. Rev. D 34, 384; E. W. Leaver, 1985 An analytic representation for the quasinormal modes of Kerr black holes, Proc. Roy. Soc. A 402, 285.

[8] S. Iyer and C. M. Will, 1987 Black-hole normal modes: A WKB approach. I. Foundations 
and application of a higher-order WKB analysis of potential-barrier scattering, Phys. Rev. D 35, 3621 ; S. Iyer, 1987 Black-hole normal modes: A WKB approach. II. Schwarzschild black holes, Phys. Rev. D 35, 3632; A. Zhidenko, 2004 Quasi-normal modes of Schwarzschild-de Sitter black holes, Class. Quant. Grav. 21, 273; R. A. Konoplya, 2003 Quasinormal behavior of the D-dimensional Schwarzschild black hole and the higher order WKB approach, Phys. Rev. D 68, 024018.

[9] H. T. Cho, A. S. Cornell, J. Doukas and W. Naylor, 2010 Black hole quasinormal modes using the asymptotic iteration method, Class. Quant. Grav. 27, 155004; C. Ma, Y. Gui, W. Wang and F. Wang, 2008 Massive scalar field quasinormal modes of a Schwarzschild black hole surrounded by quintessence, Cent. Eur. J. Phys. 6, 194.

[10] T. Roy Choudhury and T. Padmanabhan, 2004 Quasinormal modes in Schwarzschild-de Sitter spacetime: A simple derivation of the level spacing frequencies, Phys. Rev. D 69, 064033

[11] T. Padmanabhan, 2004 Quasi normal modes: A simple derivation of the level spacing of the frequencies, Class.Quant.Grav. 21 L1

[12] A. J. M Medved, D Martin and M Visser, 2004 Dirty black holes: Quasinormal modes, Class. Quant. Grav. 21, 1393.

[13] D. Batic, N. G. Kelkar and M. Nowakowski, 2011 Comment on "Quasinormal modes in Schwarzschild-de Sitter spacetime: A simple derivation of the level spacing of the frequencies", D83, 108501; T. Roy Choudhury and T. Padmanabhan, 2011 Reply to "Comment on Quasinormal modes in Schwarzschild-de Sitter spacetime: A simple derivation of the level spacing of the frequencies" Phys. Rev. D 83, 108502.

[14] R. A. Matzner and M. P. Ryan, Jr., 1997 Low-frequency limit of gravitational scattering, Phys. Rev. D16, 1636.

[15] N. G. Sanchez, 1975 Scattering of scalar waves from a Schwarzschild black hole, J. Math. Phys. 17, 688.

[16] S. R. Dolan, E. S. Oliveira and L. C. B. Crispino, 2009 Scattering of sound waves by a canonical acoustic holes, Phys. Rev. D 79, 064014.

[17] J. R. Taylor, Scattering Theory, John Wiley and Sons, 1972.

[18] V. E. Barlette, M. M. Leite and S. K. Adhikari, 2001 Integral equations of scattering in one dimension, Am. J. Phys. 69, 1010.

[19] L. C. B. Crispino, S. R. Dolan and E. S. Oliveira, 2009 Electromagnetic Wave Scattering by 
Schwarzschild Black Holes, Phys. Rev. Lett. 102, 231103.

[20] I. S. Gradshteyn and I. M. Ryhzik, Table of Integrals, Series and Products, New York: Academic Press, 1994.

[21] M. Abramowitz and I. A. Stegun, Handbook of Mathematical Functions with Formulas, Graphs, and Mathematical Tables, New York: Dover Publications, 1972.

[22] L. J. Slater, Confluent Hypergeometric Functions, Cambridge University Press, 1960.

[23] R. A. Matzner, N. Zamorano and V D Sandberg, 1979 Instability of the Cauchy horizon of Reissner-Nordström black holes, Phys. Rev. D 192821.

[24] P. Appell, 1925 Sur les fonctions hypergéometriques de plusiers variables, Gauthier-Villars, Paris, Mém. de Sciences Math. de l'Acad. de Sciences de Paris, III.

[25] B. D. Koberlein and R. L. Mallett, 1994 Charged, radiating black holes, inflation and cosmic censorship, Phys. Rev. D 495111.

[26] R. Zaho, L. C. Zhang and Z. G. Li, 1998 Hawking effect of the charged evaporating black hole in de Sitter space, Il Nuovo Cimento B 113291.

[27] R. Zhao, J. F. Zhang and L. C. Zhang, 2002 Entropy of Reissner-Nordstrom-De Sitter Black Hole in Nonthermal Equilibrium, Commun. Theor. Phys. (Beijing, China) 3745.

[28] E. Picard, 1880 Sur un extension aux fonctions de deux variables du probléme de Riemann relatif aux fonctions hypergéometriques, C. R. Acad. Sci. Paris 901119.

[29] J. Tan and P. Zhou, 2005 On the finite sum representations of the Lauricella functions $F_{D}$, Adv. Comput. Math. 23333.

[30] N. K. Kofinti, 1984 Scattering of a Klein-Gordon Particle by a Black Hole, Internat. Journ. Theoret. Physics 23, 991; F. A. Handler and R. A. Matzner, 1980 Gravitational Wave Scattering, Phys. Rev. D22, 2331.

[31] Y. Tikochinsky, 1977 Variable Amplitude Equations for One-Dimensional Scattering, Ann. Phys. 103, 185.

[32] M. G. Rozman and P. Reineker, 1994 One-dimensional scattering: Recurrence relations and differential equations for transmission and reflection amplitudes, Phys. Rev. A 49, 3310; O. Kidun, N. Fominykh and J. Berakdar, 2005 Transmission, reflection, and resonance formation in one-dimensional systems, Phys. Rev. A 71, 022703; H. Lee and Y. J. Lee, 2007 Determinable solutions for one-dimensional quantum potentials: scattering, quasi-bound and bound-state problems, J. Phys. A 40, 3569. 